\title{
Gait Retraining With Real-Time Visual Feedback to Treat Patellofemoral Pain in Adult Recreational Runners: A Critically Appraised Topic
}

\author{
Marcie Fyock, Nelson Cortes, Alex Hulse, and Joel Martin
}

\begin{abstract}
Clinical Scenario: Patellofemoral pain (PFP) is a common knee injury in recreational adult runners, possibly caused by faulty mechanics. One possible approach to reduce this pain is to retrain the runner's gait. Current research suggests that no definitive gold standard treatment for PFP exists. Gait retraining utilizing visual feedback may reduce PFP in both the short and long term. Clinical Question: In adult runners diagnosed with PFP, does gait retraining with real-time visual feedback lead to a decrease in pain? Summary of Key Findings: A literature search was performed; 3 relevant studies utilizing gait retraining with visual feedback, pain level as an outcome measure, and follow-up measures of at least 1 month after the intervention were included. All the included studies reported a decrease in short- and long-term pain for participants following visual feedback gait retraining. In addition, biomechanical measures related to PFP, including peak hip adduction angle and the angle of contralateral pelvic drop, improved after the completion of the intervention. Clinical Bottom Line: There is level 2 evidence supporting the implementation of 8 sessions over 2 weeks of visual feedback gait retraining as a means of treating patients diagnosed with PFP. Based on current available evidence, clinicians should identify faulty mechanics of patients and implement a protocol of increasing real-time visual feedback over the first 4 sessions and decreasing visual feedback over the final 4 sessions. Strength of Recommendation: Level 2.
\end{abstract}

Keywords: rehabilitation, chronic knee injury, anterior knee pain

\section{Clinical Scenario}

An estimated 20 million Americans run recreationally each year, of which $65 \%$ will incur a running-related injury yearly. ${ }^{1} \mathrm{~A}$ common complaint among this group is patellofemoral pain (PFP)—females being 2.33 times more likely to develop PFP compared with males. ${ }^{2}$ PFP is often associated with a dull to sharp pain around the patella, which worsens after ascending/descending stairs and following prolonged sitting. Up to $90 \%$ of the individuals diagnosed with PFP will report symptoms 4-18 years after their initial diagnosis. ${ }^{3}$ In addition, a history of PFP has been associated with an increased risk of developing patellofemoral joint osteoarthritis, as both conditions are associated with alterations in patella alignment and tibiofemoral alignment, further supporting the need for an effective intervention. ${ }^{4}$

Lower-extremity biomechanical factors related to running mechanics and misalignment of the lower limbs are often reported as the main causes of PFP. ${ }^{3,5-7}$ Several prominent factors appear to be related to motion of the lumbo-pelvic-hip complex. Current research suggests increased contralateral pelvic drop, hip adduction, and hip internal rotation as a potential contributing factors to the development of PFP. ${ }^{5-7}$ Strengthening the muscles of the lumbopelvic-hip complex has shown to improve knee pain ${ }^{8}$; however, this does not appear to offer consistent long-term resolution of pain. ${ }^{8}$

The purpose of this critically appraised topic (CAT) is to review the current evidence investigating real-time visual feedback as an intervention choice for the treatment of PFP in adult recreational runners.

The authors are with Sports Medicine Assessment Research and Testing, George Mason University, Manassas, VA. Fyock (mfyock@gmu.edu) is corresponding author.

\section{Focused Clinical Question}

In adult runners diagnosed with PFP, does gait retraining with realtime visual feedback lead to a decrease in pain?

\section{Summary of Search, "Best Evidence" Appraised, and Key Findings}

- The literature search identified 21 studies; 3 studies utilizing real-time visual feedback to treat PFP in adult recreational runners were included (Table 1).

- One randomized controlled trial and 2 cohort studies met the inclusion and exclusion criteria (Table 1).

- All studies utilized 8 sessions over 2-week intervention, which included education on running form, real-time visual feedback, and verbal feedback from the researchers.

- All studies increased visual feedback for the first 4 sessions, and then progressively reduced the frequency of feedback over the next 4 sessions. Verbal cues were used based on the visual feedback.

- All studies reported reduced pain outcome measures at the conclusion of the intervention and 1-month follow-up. One study included a 3-month follow-up.

- All studies reported improvement in the biomechanical measures of hip adduction angle and pelvic drop.

\section{Clinical Bottom Line}

Evidence suggests that real-time visual feedback can be implemented in a gait retraining intervention to reduce symptoms associated with PFP in adult recreational runners. ${ }^{9-11}$ Clinicians 
Table 1 Summary of Study Designs of Articles Retrieved

\begin{tabular}{llcl}
\hline $\begin{array}{l}\text { Level of } \\
\text { evidence }\end{array}$ & $\begin{array}{l}\text { Study design/ } \\
\text { methodology }\end{array}$ & $\begin{array}{c}\text { Number } \\
\text { located }\end{array}$ & References \\
\hline 2 & Randomized controlled trial & 1 & Roper et al ${ }^{9}$ \\
2 & Cohort study & 2 & $\begin{array}{l}\text { Noehren et al } \\
\text { Willy et al }^{11}\end{array}$ \\
\hline
\end{tabular}

who are experienced and knowledgeable in proper running gait mechanics may choose to perform a gait analysis on the patients to identify what aspects of gait need improving. Visual feedback using a mirror or a real-time computer display may then be used with verbal cues during retraining sessions. The included studies suggest that verbal feedback at higher frequencies over the first 4 sessions, and then gradually decreasing over the final 4 sessions, leads to positive outcomes. ${ }^{9-11}$ Significant pain reduction ${ }^{9-11}$ and improvements in self-reported function ${ }^{10,11}$ were noted in all 3 studies (Table 2). The included studies evaluated symptoms at 1month $^{9-11}$ and 3-month ${ }^{11}$ follow-up with postintervention improvements remaining. In addition, the duration of the real-time feedback intervention ( $2 \mathrm{wk}$ ) was shorter than previously reported interventions (4-12 wk) that have focused on hip and/or knee strengthening. Clinicians may choose to reevaluate patients at additional intervals to ensure that patients have maintained noted improvements. These studies suggest that providing real-time feedback of movement patterns during gait retraining may improve pain outcomes in adult runners diagnosed with PFP.

\section{Strength of Recommendation}

Grade B evidence exists to support treating the PFP in adult recreational runners with real-time visual feedback gait retraining. The Oxford Centre for Evidence-Based Medicine recommends a grade of B for consistent level 2 or 3 studies of which all of the included studies are level 2 studies.

\section{Search Strategy}

\section{Term Used to Guide Search Strategy}

- Patient: recreational adult runners

- Intervention/Assessment: gait retraining with real-time visual feedback

- Comparison: no comparison

- Outcome: symptoms associated with PFP

\section{Sources of Evidence Searched}

- PubMed

- CINAHL

- SPORTDiscus

- MEDLINE

- EBSCOhost

- Cochrane Database of Systematic Reviews

- Alt HealthWatch

- PsycINFO

- Additional resources obtained via manual search of reference lists

\section{Inclusion and Exclusion Criteria}

\section{Inclusion Criteria}

- Studies included gait retraining with visual feedback for the participants.

- Studies included adult recreational runners who were diagnosed with PFP.

- Studies included a minimum of 6 miles/wk $(10 \mathrm{~km})$ of running.

- Studies included postintervention follow-up.

- Studies included pain as outcome measure.

- Written within past 10 years (2008-2018).

- Written in the English language.

\section{Exclusion Criteria}

- Participants aged 18 years and younger or 45 years and older.

- Participants had additional lower-extremity musculoskeletal injuries or other significant health complications.

- Case studies.

\section{Results of Search}

After an extensive review of the current published literature, 21 studies were screened; in which only 3 studies met the eligibility requirements and were selected for inclusion. ${ }^{9-11}$ The studies are listed in Table 1 and summarized in Table 2. All 3 studies used realtime visual feedback as the primary intervention to treat PFP in adult recreational runners.

\section{Best Evidence}

The studies presented in Table 1 were selected for inclusion in this CAT and considered as the best available evidence. The included studies are level 2 evidence based upon the 2011 criteria from the Oxford Centre for Evidence-Based Medicine.

\section{Implications for Practice, Education, and Future Research}

Real-time feedback used to modify gait mechanics has successfully been implemented as a conservative treatment in knee pathologies such as knee osteoarthritis and joint replacement, with successful short-term outcomes. ${ }^{12,13}$ The studies included in this CAT identified effects of real-time visual feedback on pain associated with PFP in recreational runners. All of the included studies reported improvements in pain following a real-time visual feedback intervention. ${ }^{9-11}$ All 3 studies noted improvements both immediately postintervention and at 1 -month ${ }^{9,10}$ and 3 -month ${ }^{11}$ follow-up. In addition, the incorporation of real-time feedback resulted in decreases in biomechanical measures of contralateral pelvic drop, hip abduction, hip adduction, and internal rotation, suggesting the underlying mechanics associated with PFP may have changed. ${ }^{9-11}$

All studies utilized 8 retraining sessions over 2 weeks (4 sessions/wk). The running time increased over the 8 sessions, and the real-time visual feedback to the participants increased over the first 4 sessions and decreased over the last 4 sessions. In addition, all studies completed a 3-dimensional motion analysis 


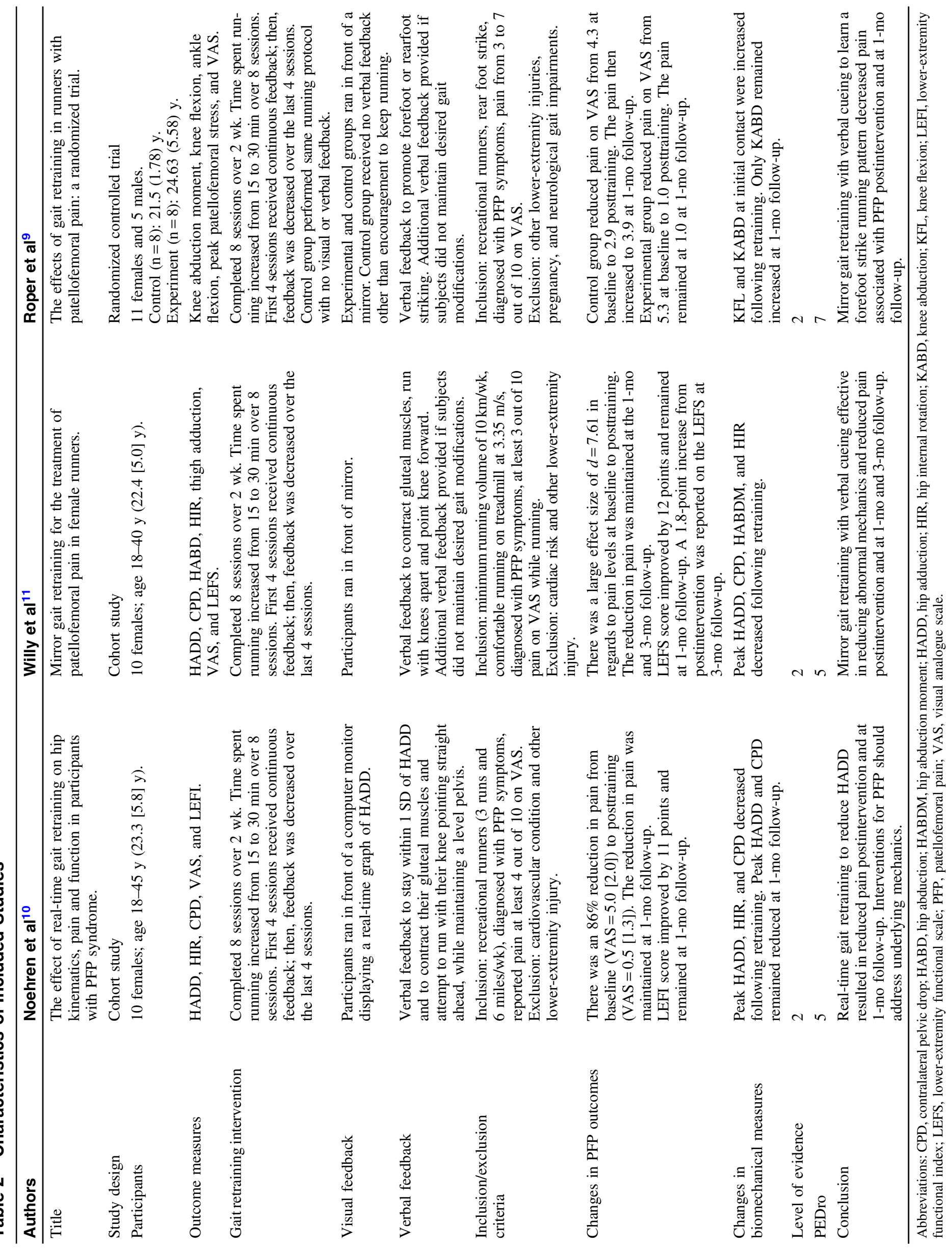


before and after gait retraining protocol for each participant. Furthermore, all studies provided some form of visual feedback for the participants, along with verbal cues and education on individualized gait patterns.

Noehren et al $^{10}$ utilized a computer display of the participants' real-time hip adduction angle on a graph generated from 3D motion software, showing $1 \mathrm{SD}$ of hip adduction mean and had the participants attempt to match the mean. In addition, participants were asked to contract their gluteal muscles and run with their knees pointed straight ahead. ${ }^{10}$ To simplify the process, Willy et al $^{11}$ modified Noehren's methodology by placing a mirror directly in front of the treadmill instead of a computer display, and which made it more applicable outside a laboratory setting. Participants received scripted instructions to run with their knees apart and kneecaps pointing straight ahead. ${ }^{11}$ Roper et $\mathrm{al}^{9}$ also utilized mirror feedback but with the goal of training the participants to run with a forefoot striking pattern, as opposed to a midfoot or rear foot strike. Scripted statements of "run on your toes" and/or "run on the balls of your feed" were used. 9 The verbal feedback provided to the subjects in the studies influenced the kinematics of the running pattern and may have contributed to the changes reported in pain and overall function.

All the studies included in this CAT utilized the visual analogue scale for measuring both immediate pain changes and retention (1-mo and 3-mo postintervention). Noehren et $\mathrm{al}^{10}$ reported an $86 \%$ reduction in pain immediately, whereas both Roper et $\mathrm{al}^{9}$ and Willy et $\mathrm{al}^{11}$ reported a significant reduction in pain postintervention (Table 2). Noehren et $\mathrm{al}^{10}$ and Roper et $\mathrm{al}^{9}$ also reported retention of pain changes 1 -month postintervention, whereas Willy et al ${ }^{11}$ noted continued improvements in pain at 3-month postintervention. These changes in pain were seen following a considerably shorter intervention ( $2 \mathrm{wk}$ ) than previously published studies that focused on hip and/or knee strengthening (4-12 wk). ${ }^{14}$ The reduction in pain reported at follow-up intervals may suggest retention of the retraining without reinforcement from the clinicians. Furthermore, the biomechanical measures related to PFP including peak hip adduction, hip internal rotation, ${ }^{10,11}$ and knee abduction angle ${ }^{9}$ improved at postintervention and remained at follow-up evaluation.

A strength of the current literature is clinicians working with patients diagnosed with PFP can emulate the overall methodology utilized by these studies. Although only a computer display ${ }^{10}$ and a mirror reflecting the participant ${ }^{9,11}$ were used for the included studies, other means of visual feedback may also be effective, such as a real-time video feed of the patient's running gait. Furthermore, increasing and then decreasing the amount of feedback and verbal cues over the sessions allow the participant to internalize and reinforce the retraining. ${ }^{11}$ Clinicians may choose to individualize the frequency and amount of feedback based on client needs and performance. Individuals may move through the 3 stages of motor learning (cognitive, associative, and autonomous) at different rates; thus, the intervention may need to be adjusted accordingly. ${ }^{15}$ Movements in the cognitive stage are characterized as slower, greater variability, less efficient, and requiring considerable cognitive activity to perform. The associative stage occurs when movement becomes more fluid and efficient. Less cognitive activity is needed to perform the movement; however, individuals must still devote conscious attentional focus to perform the movement. The final stage of motor learning, autonomous, is characterized by highly fluid, efficient, and accurate movement with control of movement being relatively automatic. Clinicians should consider the stage of motor learning a patient is in when providing feedback to a patient.
Limitations existed in all the studies. Two studies included only female participants, ${ }^{10,11}$ whereas Roper et $\mathrm{al}^{9}$ studied 11 female and 5 male participants. Although female runners are reported to have a higher reported rate of PFP than corresponding males due to their increased hip adduction, ${ }^{10}$ the conclusions of the studies are limited concerning treatment for male runners. As men tend to naturally have lower hip adduction angles than women, interventions should emulate the methodology of Roper et al, ${ }^{9}$ which addressed foot strike instead of hip angles. A second limitation was the verbal cues provided to subjects, which may have resulted in an internal attention focus. An internal attention focus is when one's attention is directed to locations inside the body, or to motor or sensory information. Alternatively, an external attention focus can be defined as attention directed outside the body to an object or performance goal. Research on motor learning has shown that an external focus of attention enhances motor performance and learning. ${ }^{15}$ The verbal cues used in all studies directed the attentional focus of subjects to specific muscles and body parts. ${ }^{9-11}$ This may have led subjects to adopt an internal focus of attention, which has been shown to be less effective for motor learning of various tasks than instructions promoting an external focus of attention. ${ }^{15}$ In addition, the use of a mirror for visual feedback in performance of a single-joint and multijoint tasks is likely not as efficacious as verbal instructions leading to an external focus of attention. ${ }^{16}$

The authors would like to offer several suggestions to improve knowledge related to the implementation of real-time visual feedback as an intervention choice for PFP in adult runners. First, future studies should include long-term follow-up with participants to assess the lasting efficacy of the intervention. Second, future studies should modify the intervention methodology concerning its frequency, duration, and attentional focus of verbal cues. Not all clinicians are able to schedule 8 treatment sessions over the course of 2 weeks; researchers may choose to investigate the efficacy of intervention sessions that are weekly in nature. Finally, this CAT should be reviewed in 2 years to determine whether additional best research evidence has been published that could aid in answering the focused clinical question.

\section{References}

1. Messier SP, Martin DF, Mihalko SL, et al. A 2-year prospective cohort study of overuse running injuries: the Runners and Injury Longitudinal Study (TRAILS). Am J Sports Med. 2018;46(9):22112221. PubMed ID: 29791183 doi:10.1177/0363546518773755

2. Boling M, Padua D, Marshall S, Guskiewicz K, Pyne S, Beutler A. Gender differences in the incidence and prevalence of patellofemoral pain syndrome. Scand J Med Sci Sports. 2010;20(5):725-730. PubMed ID: 19765240 doi:10.1111/j.1600-0838.2009.00996.x

3. Neal BS, Barton CJ, Gallie R, O'Halloran P, Morrissey D. Runners with patellofemoral pain have altered biomechanics which targeted interventions can modify: a systematic review and meta-analysis. Gait Posture. 2016;45:69-82. PubMed ID: 26979886 doi:10.1016/j. gaitpost.2015.11.018

4. Hinman RS, Lentzos J, Vicenzino B, Crossley KM. Is patellofemoral osteoarthritis common in middle-aged people with chronic patellofemoral pain? Arthritis Care Res. 2014;66(8):1252-1257. doi:10. 1002/acr.22274

5. Barton CJ, Levinger P, Menz HB, Webster KE. Kinematic gait characteristics associated with patellofemoral pain syndrome: a systematic review. Gait Posture. 2009;30(4):405-416. PubMed ID: 19651515 doi:10.1016/j.gaitpost.2009.07.109 
6. Fox A, Ferber R, Saunders N, Osis S, Bonacci J. Gait kinematics in individuals with acute and chronic patellofemoral pain. Med Sci Sports Exerc. 2018;50(3):502-509. PubMed ID: 29077638 doi:10. 1249/MSS.0000000000001465

7. Dierks TA, Manal KT, Hamill J, Davis IS. Proximal and distal influences on hip and knee kinematics in runners with patellofemoral pain during a prolonged run. J Orthop Sports Phys Ther. 2008;38(8):448-456. PubMed ID: 18678957 doi:10.2519/jospt. 2008.2490

8. Fukuda TY, Melo WP, Zaffalon BM, et al. Hip posterolateral musculature strengthening in sedentary women with patellofemoral pain syndrome: a randomized controlled clinical trial with 1-year follow-up. J Orthop Sports Phys Ther. 2012;42(10):823-830. PubMed ID: 22951491 doi:10.2519/jospt.2012.4184

9. Roper JL, Harding EM, Doerfler D, et al. The effects of gait retraining in runners with patellofemoral pain: a randomized trial. Clin Biomech Bristol Avon. 2016;35:14-22. doi:10.1016/j.clinbiomech.2016.03. 010

10. Noehren B, Scholz J, Davis I. The effect of real-time gait retraining on hip kinematics, pain and function in subjects with patellofemoral pain syndrome. Br J Sports Med. 2011;45(9):691-696. PubMed ID: 20584755 doi:10.1136/bjsm.2009.069112
11. Willy RW, Scholz JP, Davis IS. Mirror gait retraining for the treatment of patellofemoral pain in female runners. Clin Biomech Bristol Avon. 2012;27(10):1045-1051. doi:10.1016/j.clinbiomech. 2012.07.011

12. Agresta C, Brown A. Gait retraining for injured and healthy runners using augmented feedback: a systematic literature review. J Orthop Sports Phys Ther. 2015;45(8):576-584. PubMed ID: 26158882 doi:10.2519/jospt.2015.5823

13. Eddo O, Lindsey B, Caswell SV, Cortes N. Current evidence of gait modification with real-time biofeedback to alter kinetic, temporospatial, and function-related outcomes: a review. Int J Kinesiol Sports Sci. 2017;5(3):35-55. doi:10.7575/aiac.ijkss.v.5n.3p.35

14. Clijsen R, Fuchs J, Taeymans J. Effectiveness of exercise therapy in treatment of patients with patellofemoral pain syndrome: systematic review and meta-analysis. Phys Ther. 2014;94:1697-1708. PubMed ID: 25082920 doi:10.2522/ptj.20130310

15. Wulf G. Attention and Motor Skill Learning. Champaign, IL: Human Kinetics; 2007.

16. Halperin I, Hughes S, Panchuk D, Abbiss C, Chapman DW. The effects of either a mirror, internal or external focus instructions on single and multi-joint tasks. PLoS One. 2016;11(11):e0166799. doi:10.1371/journal.pone.0166799 